\title{
On the Response of a Micro Non-Destructive Testing X-ray Detector
}

\author{
Dionysios Linardatos ${ }^{1}{ }^{\mathbb{D}}$, Vaia Koukou ${ }^{1}$, Niki Martini ${ }^{1}$, Anastasios Konstantinidis ${ }^{2}$, Athanasios Bakas ${ }^{3}$, \\ George Fountos ${ }^{1}$, Ioannis Valais ${ }^{1, *(D)}$ and Christos Michail ${ }^{1}$ (D)
}

1 Radiation Physics, Materials Technology and Biomedical Imaging Laboratory, Department of Biomedical Engineering, University of West Attica, Ag. Spyridonos, 12210 Athens, Greece; dlinardatos@uniwa.gr (D.L.); koukou@uniwa.gr (V.K.); mmartini@uniwa.gr (N.M.); gfoun@uniwa.gr (G.F.); cmichail@uniwa.gr (C.M.)

2 Radiological Sciences Group, Department of Medical Physics, Queen Alexandra Hospital, Portsmouth Hospitals University NHS Trust, Portsmouth PO6 3LY, UK; Anastasios.Konstantinidis@porthosp.nhs.uk

3 Department of Biomedical Sciences, University of West Attica, Ag. Spyridonos, 12210 Athens, Greece; abakas@uniwa.gr

* Correspondence: valais@uniwa.gr; Tel.: +30-210-5385-371

check for updates

Citation: Linardatos, D.; Koukou, V.; Martini, N.; Konstantinidis, A.; Bakas A.; Fountos, G.; Valais, I.; Michail, C. On the Response of a Micro

Non-Destructive Testing X-ray Detector. Materials 2021, 14, 888. https://doi.org/10.3390/ma14040888

Academic Editor: Giovanni Bruno

Received: 7 January 2021

Accepted: 10 February 2021

Published: 13 February 2021

Publisher's Note: MDPI stays neutral with regard to jurisdictional claims in published maps and institutional affiliations.

Copyright: (c) 2021 by the authors. Licensee MDPI, Basel, Switzerland. This article is an open access article distributed under the terms and conditions of the Creative Commons Attribution (CC BY) license (https:/ / creativecommons.org/licenses/by/ $4.0 /)$.

\begin{abstract}
Certain imaging performance metrics are examined for a state-of-the-art $20 \mu \mathrm{m}$ pixel pitch CMOS sensor (RadEye HR), coupled to a $\mathrm{Gd}_{2} \mathrm{O}_{2} \mathrm{~S}: \mathrm{Tb}$ scintillator screen. The signal transfer property (STP), the modulation transfer function (MTF), the normalized noise power spectrum (NNPS) and the detective quantum efficiency (DQE) were estimated according to the IEC 62220-1-1:2015 standard. The detector exhibits excellent linearity (coefficient of determination of the STP linear regression fit, $\mathrm{R}^{2}$ was 0.9978 ), while its DQE peaks at $33 \%$ and reaches $10 \%$ at a spatial frequency of 3 cycles $/ \mathrm{mm}$, for the measured with a Piranha RTI dosimeter (coefficient of variation CV $=0.03 \%$ ) exposure value of $28.1 \mu \mathrm{Gy}$ DAK (detector Air Kerma). The resolution capabilities of the X-ray detector under investigation were compared to other commercial CMOS sensors, and were found in every case higher, except from the previous RadEye HR model (CMOS- $\mathrm{Gd}_{2} \mathrm{O}_{2} \mathrm{~S}$ : Tb screen pair with $22.5 \mu \mathrm{m}$ pixel pitch) version which had slightly better MTF. The present digital imager is designed for industrial inspection applications, nonetheless its applicability to medical imaging, as well as dual-energy is considered and certain approaches are discussed in this respect.
\end{abstract}

Keywords: CMOS; imaging; $\mathrm{Gd}_{2} \mathrm{O}_{2} \mathrm{~S}$ :Tb; ZnSe:Te; non-destructive testing; DQE; scintillators; IEC 62220-1-1:2015

\section{Introduction}

The last decades have witnessed an unprecedented growth in the development of X-ray digital imaging technologies, to the extent that it is yet difficult to imagine the modern radiology in their absence. Digital imagers have revolutionized the otherwise classic procedures such as radiography, but more importantly have provided advanced techniques like digital breast tomosynthesis (DBT) [1-3], digital subtraction angiography (DSA) [4], thin tissue autoradiography [5,6], X-ray phase contrast imaging (XPCi) [7], etc. In the industrial field, this technology finds application in non-destructive testing (NDT) as well. In this quality control method, real-time radiography (RTR) is used for projection imaging and inspection of components from a production line. Material characterization and evaluation of substance properties are further applications under the term non-invasive inspection techniques. X-ray refraction, for instance, utilizes digital imagers for the characterization of well-defined geometry samples (fibers, capillaries) or bulks of micro-particles [8-10].

Digital imagers in their indirect-detection version, i.e., coupled to a scintillator material, have turned up in various forms; amorphous silicon on thin-film-transistor panels (a-Si-TFT) and their high-purity counterparts, charge-coupled devices (CCD) and complementary metal-oxide semiconductors (CMOS), either active or passive pixel sensor (APS or PPS) [11,12]. 
CCD detectors exhibit high resolution, good response linearity, low dark signal, low read noise and high sensitivity, making them superior at low exposures [12,13]. Initially their active surface used to be limited to less than $7 \mathrm{~cm}^{2}$ [14], but today's designs have reached $85 \mathrm{~cm}^{2}$ [15], albeit at a high cost. Those small-sized CCDs necessitate demagnification solutions for applications that require larger fields of view [16]. This inserts an additional source of noise and degrades image quality, up to the point of determining the whole system's performance (quantum sink) $[11,17,18]$. Something which, along with the specialized manufacturing process that these devices demand, adds to the complexity and gives rise to the overall cost. Moreover, CCDs are prone to radiation damage $[19,20]$.

CMOS imagers take advantage of their sharing a highly developed fabrication process with the semiconductor industry, a field that progresses in a frantic pace and continuously curtails costs. Hence, they are presently a cost-effective solution that offers very high frame rates, $X-Y$ addressability and low power consumption [21]. Their detecting area can reach more than $160 \mathrm{~cm}^{2}$, while they can be designed 2-3 sides buttable [2,14], allowing placement in a tiled fashion and the building of a large area imager. The need for the (detrimental for the image quality) demagnification is eliminated and a direct coupling of the scintillator material is thus permitted. Regarding the attainable resolution, sub $-2 \mu \mathrm{m}$ pixel sizes have been reported [22,23].

Initially, CMOS's main disadvantage over CCD was the higher electronic noise [24]. Today's APS designs, by integrating advanced functions like buffering and amplification on-chip and in-pixel, outperform in this respect. At the same time, they can support much higher read-out speeds because of their massively parallel architecture $[7,25]$, that can be obtained, along with lower bandwidth-lower noise ADCs (analog to digital converters). Frame rates above $600 \mathrm{fps}$ (frames per second) have been achieved for a general-purpose imager [26], while speeds of the order of $\mathrm{MHz}$ (Megahertz) have been realized using pixel-based storage for an ultra-high speed (UHS) one [27].

Apparently, certain of the novel medical imaging applications of the digital imagers can benefit from the distinguishable merits of the CMOS detectors. For example, a digital mammography detector has to be large enough for full field coverage, as well as fast enough in acquiring images, without lag and baseline drifts [21,28]. Similarly, in the industrial field, requirements include high resolution, physical ruggedness, high frame rates, cost effectiveness and portability for in-field applications. All the specifications above can be met by CMOS technology [10].

The aim of the present study was to examine the imaging performance of a state-ofthe-art indirect detection CMOS X-ray detector for imaging applications, such as dualenergy (DE), in which the small pixel size could be beneficial in the detection of micro calcifications and masses, indicative of breast cancer, as well as industrial inspection applications requiring increased resolution to detect sub-millimeter structures [29]. Imaging performance metrics, such as the signal transfer property (STP), the modulation transfer function (MTF) and the normalized noise power spectrum (NNPS) were measured, to finally estimate the detective quantum efficiency (DQE) of the imaging system, following the International Electrotechnical Commission (IEC) procedures.

\section{Materials and Methods}

The detector under investigation is the Remote RadEye HR CMOS (Teledyne DALSA, Waterloo, ON, Canada) [30] photodiode pixel array (N-well diffusion on p-type epitaxial silicon), that features an active area of $33.0 \times 24.9 \mathrm{~mm}^{2}$, with $1650 \times 1246$ pixels at $20 \mu \mathrm{m}$ pitch. The detector can be used in industrial applications, since it is small-sized and can reach tight spaces with its 12-bit USB interface. The Carestream Min-R 2190 scintillator screen (gadolinium oxysulfide activated with terbium: $\mathrm{Gd}_{2} \mathrm{O}_{2} \mathrm{~S}$ : Tb of thickness $85 \mu \mathrm{m}$ and screen coating weight of $33.91 \mathrm{mg} / \mathrm{cm}^{2}$ ) is in direct contact with the CMOS active area, while a carbon-fiber window shields from the ambient light and provides mechanical protection $[30,31]$. 
The nominal conversion gain of the detector is 155 electrons per pixel value ( $\left.\mathrm{e}^{-} / \mathrm{PV}\right)$. The nominal electronic noise (root mean square; r.m.s.) is around $310 \mathrm{e}^{-}$and the dynamic range is $66 \mathrm{~dB}$. Hence, the calculated saturation charge is around $620 \times 10^{3} \mathrm{e}^{-}$. The maximum frame rate is $0.7 \mathrm{fps}$ and the nominal average dark current is $930 \mathrm{e}^{-} / \mathrm{s}$ at $23{ }^{\circ} \mathrm{C}$. A two-meter shielded cable is included to connect the sensor head to the electronics module, where the analog video signal is processed, digitized using 12-bit ADCs and transferred to a PC [30]. The previous model of this CMOS X-ray detector was studied by Konstantinidis [32], with $60 \mathrm{e}^{-} / \mathrm{PV}$ conversion gain (with a high gain option $2 \times$ available), $120 \mathrm{e}^{-}$ electronic noise (r.m.s.), $72 \mathrm{~dB}$ dynamic range, $0.5 \mathrm{fps}$ maximum frame rate, $3000 \mathrm{e}^{-} / \mathrm{s}$ average dark current (at $23^{\circ} \mathrm{C}$ ), one meter shielded cable and 12 bit-depth ADC [32].

Due to the variety of digital imaging detector configurations (sensors and scintillators combinations) it is necessary to establish standard methods to facilitate intercomparisons between various systems, in order to juxtapose their overall performance, in terms of widely used imaging metrics, such as the MTF, NNPS and DQE [33]. To this aim, the International Electrotechnical Commission (IEC) has established a standard method (IEC 62220-1:2003 [34]; referred to as IEC 2003 for brevity) which was amended in 2015 (IEC 62220-1-1:2015 [35]; also referred to as IEC 2015). The modifications between the two protocols were examined previously in [33]. In the present work, these metrics were estimated following the 2015 version, except for the MTF that was calculated additionally according to the 2003 version, in order to compare with the MTF values provided in the manufacturer's datasheet. The COQ plugin (Verison 2.6) for the ImageJ suite (Version 1.52a) [X]was used for the MTF, NNPS, DQE calculations [36,37].

The Del Medical Eureka radiographic system (Harisson, NY, USA) was used for the experiments. It features a rotating tungsten (W) anode with a focal spot size of $0.6 \mathrm{~mm}$ ("small" focal spot size selected) and an inherent filtration equivalent to $3 \mathrm{~mm}$ aluminum (Al). The RQA-5 radiation quality was used throughout the experiments, i.e., $70 \mathrm{kVp}$ tube voltage and $6.8 \mathrm{~mm}$ half value layer (HVL). It was found that, in addition to the tube's inherent filtration, another $21 \mathrm{~mm} \mathrm{Al} \mathrm{(type} \mathrm{1100,} \mathrm{purity} \mathrm{99 \% )} \mathrm{must} \mathrm{be} \mathrm{added} \mathrm{in} \mathrm{order} \mathrm{to}$ achieve this HVL. The Al filter was placed as close to the tube as possible and the source to detector distance (SDD) was $156 \mathrm{~cm}$.

The STP or X-ray sensitivity of the detector is the relationship between the mean pixel value (MPV) and Air Kerma at Detector's surface (DAK). To obtain the DAK, the CMOS was removed altogether, and a calibrated RTI Piranha X-ray dosimeter (Mölndal, Sweden) was placed at the same position. In line with the IEC standard's recommendations regarding the reduction of backscatter to $<0.5 \%$, a $4 \mathrm{~mm}$ thick lead foil was placed at $45 \mathrm{~cm}$ behind the dosimeter [35]. A sequence of flat-field images was acquired at seven different exposure levels, each one consisting of five repetitions, for averaging reasons. The MPV was sampled from a $1 \times 1 \mathrm{~cm}^{2}$ region of interest (ROI) and the system's response curve was fitted using a linear equation of the form:

$$
\mathrm{MPV}=\mathrm{a} \mathrm{K}_{\mathrm{a}}+\mathrm{b}
$$

where $a$ is the detector's gain factor $(G)$ [38] and $b$ is the pixel offset at zero DAK [39].

The MTF of the detector is the variation of the output contrast as a function of the spatial frequency, and is normalized to the input contrast [40,41]. Following the IEC standard's procedures [34,35], the MTF was measured using the slanted edge technique with the PTW Freiburg tungsten edge device, which consists of a $1 \mathrm{~mm}$ thick tungsten edge plate $\left(100 \times 75 \mathrm{~mm}^{2}\right)$ fixed on a $3 \mathrm{~mm}$ thick lead plate $[33,42]$. The edge device was placed in contact with the detector's entrance window at a shallow angle $\left(1.5^{\circ}-3^{\circ}\right)$ with respect to the pixel rows or columns. Images were obtained at $28.1 \mu \mathrm{Gy}$ and $48.3 \mu \mathrm{Gy}$ exposures.

For the edge spread function (ESF), a $2 \times 2 \mathrm{~cm}^{2}$ ROI was drawn, with the edge roughly at the center. The ROI's size, smaller than the suggested by the IEC protocol, is mandated by the detector's size. According to the IEC 62220-1:2003, the final MTF is determined by averaging the multiple MTFs obtained from the individual groups of $\mathrm{N}$ consecutive lines along the edge [34]. On the other hand, in the IEC 62220-1-1:2015 the final MTF is 
obtained by averaging the oversampled ESFs [35,43], by fitting of a modified Fermi-Dirac (F-D) distribution function of the form:

$$
\operatorname{Fermi}(x)=\left(\frac{c}{e^{\frac{x-a}{b}}+1}\right)+d
$$

The oversampled ESFs resulted from the pixel values of the linearized data (using the inverse of the STP curve to get DAK values) of $\mathrm{N}$ consecutive lines across the edge. With differentiation of the fitted ESF, the line spread function (LSF) is obtained which, after the application of a Hanning filter (window width 2048 pixels) is Fourier-transformed to provide the MTF [35].

The NPS of the detector expresses the statistical variance of the output signal as a function of the spatial frequency. It was determined using flat-field images at the two exposure levels ( $28.1 \mu \mathrm{Gy}$ and $48.3 \mu \mathrm{Gy}$ coefficient of variation $\mathrm{CV}=0.03 \%$ ) measured with the RTI Piranha X-ray dosimeter. The normalized noise power spectrum (NNPS) tends to provide an estimation of the output noise, independent of gross exposure variations over the detector area (e.g., heel effect) [41,44]. This is achieved by fitting and then subtracting a two dimensional (2-D) second order polynomial fit $S(x, y)$ to the original image data $I(x, y)$ after converting to $K_{a}$ units (see linearization process), using the inverse of the STP linear equation [35]. The average 2-D NPS is given by:

$$
\operatorname{NPS}(\mathrm{u}, \mathrm{v})=\frac{\Delta \mathrm{x} \Delta \mathrm{y}}{\mathrm{MN}_{\mathrm{x}} \mathrm{N}_{\mathrm{y}}} \sum_{\mathrm{m}=1}^{\mathrm{M}}\left|\operatorname{FFT}\left\{\mathrm{I}\left(\mathrm{x}_{\mathrm{i}}, \mathrm{y}_{\mathrm{i}}\right)-\mathrm{S}\left(\mathrm{x}_{\mathrm{i}}, \mathrm{y}_{\mathrm{i}}\right)\right\}\right|^{2}
$$

where $\mathrm{u}$ and $\mathrm{v}$ denote the $\mathrm{x}$ and $\mathrm{y}$-axis spatial frequency, respectively; $\Delta \mathrm{x}$ and $\Delta \mathrm{y}$ are the $\mathrm{x}$ and $\mathrm{y}$-axis pixel pitches; $\mathrm{N}_{\mathrm{x}}$ and $\mathrm{N}_{\mathrm{y}}$ express the ROI size in the $\mathrm{x}$ and $\mathrm{y}$ axes (256 pixels according to the IEC); $\mathrm{M}$ is the number of ROIs used in the ensemble average; FFT denotes the fast Fourier transform operation [2].

From this, the horizontal and vertical NPS were extracted, by averaging seven rows and seven columns on each side of each axis. Axes themselves were excluded, as the IEC standard suggests, since they may contain remnant column-to-column and/or row-to-row fixed-pattern noise (FPN). These 1-D NPS were divided by the square of the averaged $\mathrm{K}_{\mathrm{a}}[33,45]$, also known as large area signal, in order to obtain the NNPS in both orientations, which were then combined to obtain the radial average NNPS.

The detector's DQE expresses its efficiency in transferring the signal to noise ratio square $\left(\mathrm{SNR}^{2}\right)$ impinging on it, towards the output $[41,46]$. It is given by:

$$
\operatorname{DQE}(\mathrm{u})=\frac{\operatorname{MTF}^{2}(\mathrm{u})}{\mathrm{K}_{\mathrm{a}} \cdot \mathrm{q} \cdot \operatorname{NNPS}(\mathrm{u})}
$$

where $\mathrm{q}$ is the fluence per Air Kerma ratio, i.e., the number of X-ray photons per unit Air Kerma (in $\mu$ Gy) per mm $^{2}$. According to the IEC 62220-1-1:2015 protocol, the value of 29653 was used for the utilized X-ray beam quality (RQA-5) [35].

\section{Results and Discussion}

The MPV as a function of DAK is drawn in Figure 1 for the seven exposure levels and a linear interpolation is calculated. 


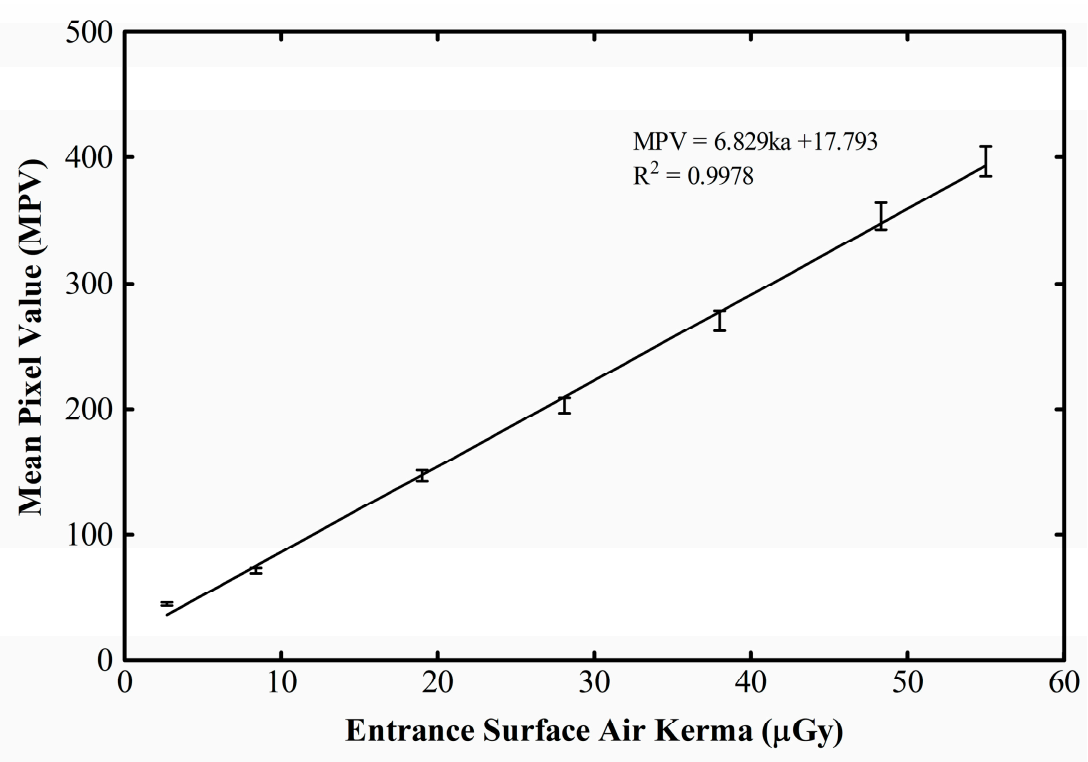

Figure 1. STP curve of the CMOS X-ray detector.

The detector's excellent linearity in the examined exposure range is obvious, since the coefficient of determination $\left(R^{2}\right)$ is 0.9978 . The gain factor $(G)$ is found 6.829 digital units (DU) per $\mu \mathrm{Gy}$. It is a higher value compared to the 5.487 measured for the previous (discontinued) version of the Remote RadEye HR detector, studied by Konstantinidis [32], at almost the same beam quality $(74 \mathrm{kVp})$. It is higher as well, in comparison to the 3.829 gain at $52 \mathrm{kVp}$, of the same study [32]. In that situation, the detector was also coupled to a $\mathrm{Gd}_{2} \mathrm{O}_{2} \mathrm{~S}: \mathrm{Tb}$ screen of mass thickness $33.91 \mathrm{mg} / \mathrm{cm}^{2}$. The dynamic range of the present (new version) detector is practically double (up to $55 \mu \mathrm{Gy}$, as opposed to $28 \mu \mathrm{Gy}$ of the old one), even though no loss of linearity is observed up to the exposure maxima in any case. There is also an offset of $\sim 18 \mathrm{DU}$ at zero input.

Despite the fact that two different detectors are being considered, each one with its own characteristics, increased $\mathrm{G}$ with increasing mean $\mathrm{X}$-ray energy is an expected behavior. As described by Marshall [47] and Konstantinidis [2], with increasing mean energy of the spectrum, the number of X-ray photons per unit DAK increases as well. Every X-ray photon of higher energy causes the emission of more optical photons by the scintillator. Furthermore, higher energy $\mathrm{X}$-ray photons have a greater penetration within the scintillator material, shifting the depth of interaction closer to the CMOS optical sensor and improving the detection probability of the secondary generated optical photons.

The oversampled ESF as a function of position across the edge device (in terms of number of pixels) is depicted in Figure 2a at $28.1 \mu \mathrm{Gy}$ and in Figure $3 \mathrm{a}$ at $48.3 \mu \mathrm{Gy}$. Figures $2 b$ and $3 b$ show the corresponding averaged and Fermi-fitted ESFs. Signal fluctuations are mostly prominent in the area where the beam is not attenuated by the edge device, due to the Poisson distribution of input X-rays in spatial and temporal domains. Contrarily, in the "dark" area the fluctuations are mostly due to the electronic noise. The resulting LSFs after the fast Fourier transform (FFT) are demonstrated in Figures $2 c$ and $3 c$, respectively, while the MTF curves according to the IEC 62220-1-1:2015 standard are shown in Figures $2 \mathrm{~d}$ and $3 \mathrm{~d}$, respectively. 
(a)
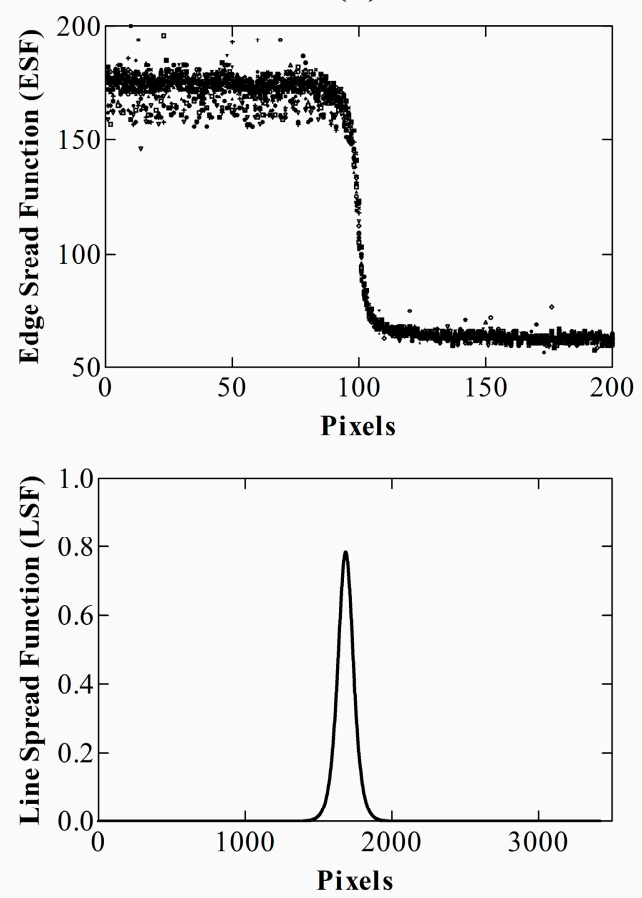

(c)
$28.1 \mu \mathrm{Gy}$

(b)
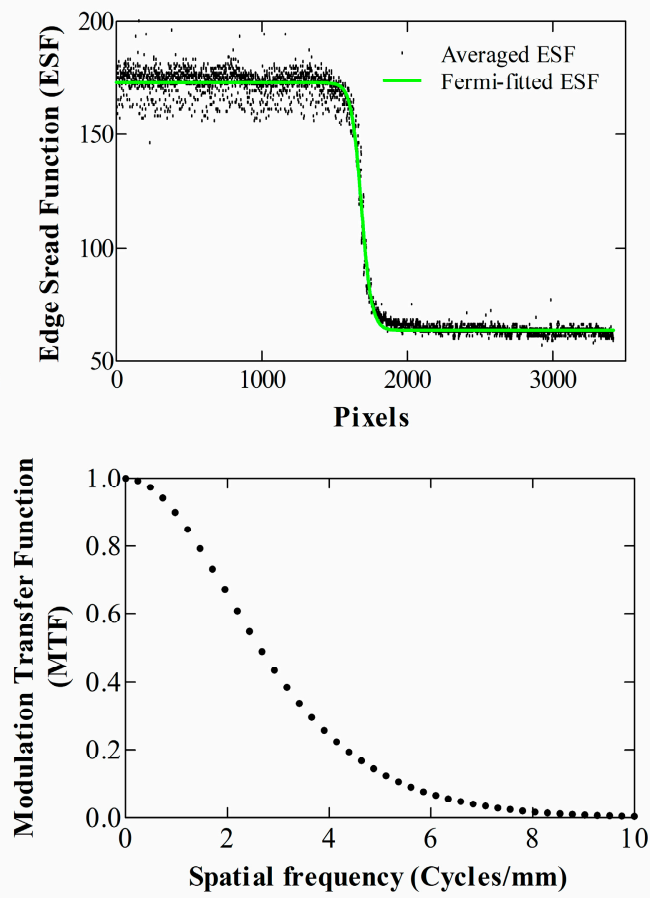

(d)

Figure 2. MTF calculation steps at an exposure level of $28.1 \mu \mathrm{Gy}$, based on the IEC 2015; (a) all ESFs; (b) averaged and Fermi-fitted ESF; (c) LSF; (d) MTF.

(a)
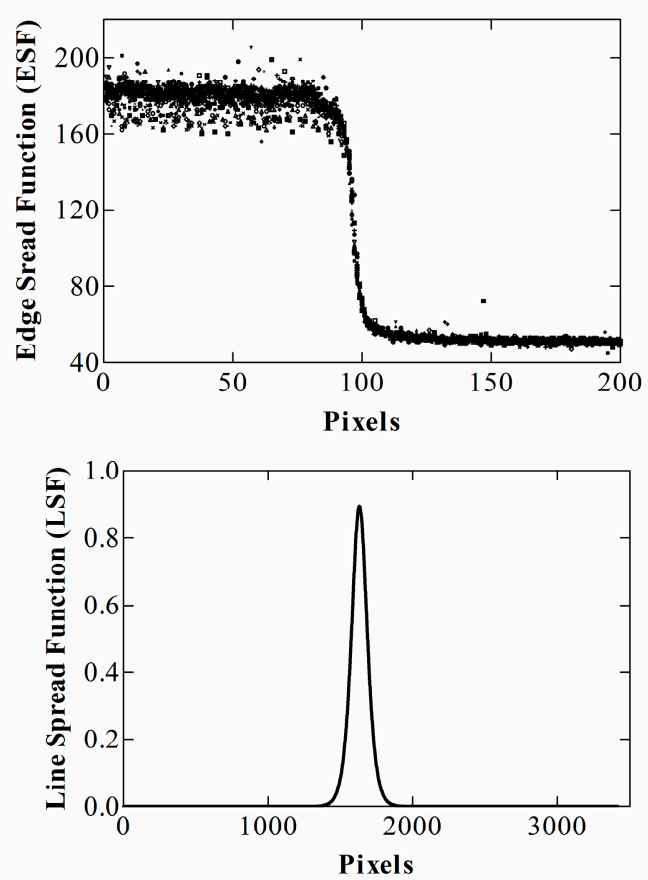

(c)
$48.3 \mu \mathrm{Gy} \quad$ (b)
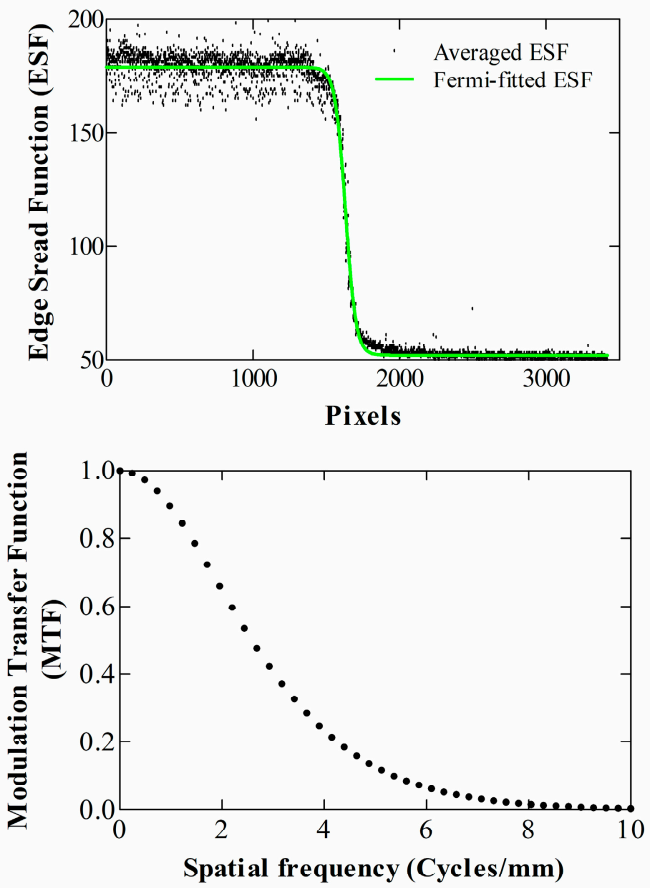

(d)

Figure 3. MTF calculation steps at an exposure level of $48.3 \mu \mathrm{Gy}$, based on the IEC 2015; (a) all ESFs; (b) averaged and Fermi-fitted ESF; (c) LSF; (d) MTF. 
For ease of comparison, these two MTF curves, along with the two MTFs according to the IEC 62220-1:2003 standard and the one extracted from the detector's datasheet, also calculated with the 2003 protocol [30], are illustrated in Figure 4. Regarding the two IEC 62220-1-1:2015 curves, there is a complete agreement between the different exposure levels. As for the two IEC 62220-1:2003 curves, the differences are within 4\%; apart from the 12\% at 10 cycles $/ \mathrm{mm}$. Hence, regardless of the exposure value, the MTFs of the 2003 and the 2015 versions of the IEC protocol show an agreement within $10 \%$, up to the 3 cycles $/ \mathrm{mm}$. After that spatial frequency they start to diverge, with the curves originating from the 2003 protocol lying higher (up to $\sim 17 \%$ difference roughly in the range $4.5-7$ cycles $/ \mathrm{mm}$ ). This difference should be attributed to the averaging method of the 2003 protocol, which influences the F-D fitting and leads to an overestimation of the MTF [33]. On the other hand, the MTF curve extracted from the detector's datasheet follows within $6 \%$ agreement the two IEC 62220-1:2003 curves, except for the 13\% difference to the $28.1 \mu$ Gy curve at 10 cycles/mm. Presumably, an algorithm close to the IEC 2003 standard was used for the datasheet MTF curve calculation.

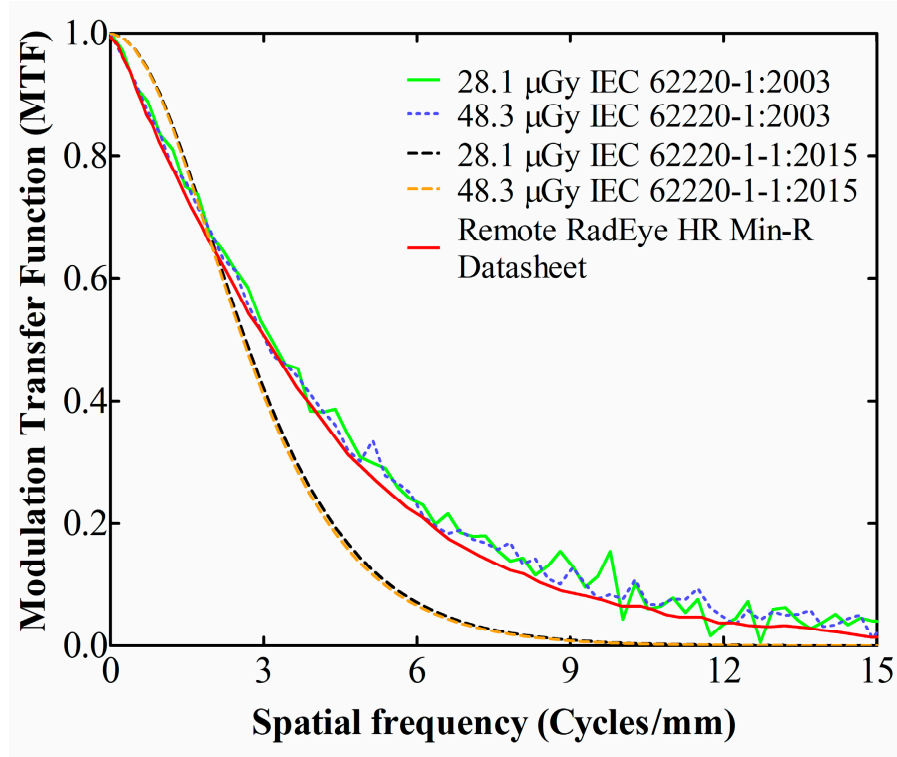

Figure 4. MTF comparison between measured values according to IEC 2003 and IEC 2015, along with manufacturer data [30].

Taking the IEC 62220-1-1:2015 MTF curve as a reference, it is noted that it falls to 50\% (MTF50) at 2.6 cycles $/ \mathrm{mm}$ and to $10 \%$ (MTF10) at 5.4 cycles $/ \mathrm{mm}$, which marks the limiting resolution of the detector [48].

For comparison purposes, the MTF values at $50 \%$ and $10 \%$ of the examined detector $[49,50]$ are shown in the Table 1 (1st column), along with the corresponding values of other commercial detectors, all calculated following the IEC 2003 standard. In the 2nd column, the previous (discontinued) version of the Remote RadEye HR CMOS photodiode array is shown, with an active area of $2.7 \times 3.6 \mathrm{~cm}^{2}$, manufactured by Rad-icon Imaging Corp. (USA) with $1200 \times 1600$ pixels and a pixel pitch of $22.5 \mu \mathrm{m}$ [32]. In the 3rd and 4th columns, the Dexela flat panel CMOS X-ray detector with a pixel pitch of $74.8 \mu \mathrm{m}$ and $1944 \times 1536$ pixels, resulting in a $14.5 \times 11.5 \mathrm{~cm}^{2}$ active area. It is configured with two different Cesium Iodide (CsI) scintillator screens, with thicknesses 150 and $600 \mu \mathrm{m}$, respectively. In the 5th column the Hamamatsu C9732DK, which is a CMOS X-ray detector with $2400 \times 2400$ pixels and $50 \mu \mathrm{m}$ pixel pitch, corresponding to an active photodiode area of $12 \times 12 \mathrm{~cm}^{2}$ [51]. Finally, in the 6th column, the Large Area Sensor (LAS) that contains $1350 \times 1350$ pixels at $40 \mu \mathrm{m}$ pitch (photodiode area $5.4 \times 5.4 \mathrm{~cm}^{2}$ ) [52]. As it can be seen from Table 1, the RadEye HR, in both versions, retained the highest MTF values due to the smallest pixel pitch and the gold standard $85 \mu \mathrm{m} \mathrm{Gd}_{2} \mathrm{O}_{2} \mathrm{~S}$ : Tb screen. However, the new 
RadEye HR version appears to have slightly worse MTF than the previous version $(4.8 \%$ and $5.5 \%$ differences at $10 \%$ and $50 \%$ of the MTF, respectively).

Table 1. MTF values at $10 \%$ and $50 \%$ for various CMOS X-ray detectors [32].

\begin{tabular}{|c|c|c|c|c|c|c|}
\hline Detector & $\begin{array}{c}\text { RadEye HR } \\
20.0 \mu \mathrm{m} \text { Pitch }\end{array}$ & $\begin{array}{c}\text { RadEye HR } \\
22.5 \mu \mathrm{m} \text { Pitch }\end{array}$ & $\begin{array}{c}\text { Dexela } \\
150 \mu \mathrm{m} \mathrm{CsI}\end{array}$ & $\begin{array}{c}\text { Dexela } \\
600 \mu \mathrm{m} \mathrm{CsI}\end{array}$ & $\begin{array}{c}\text { Hamamatsu } \\
\text { C9732DK }\end{array}$ & LAS \\
\hline Pixel Pitch $(\mu \mathrm{m})$ & 20.0 & 22.5 & 74.8 & 74.8 & 50 & 40 \\
\hline Dimensions (pixels) & $1650 \times 1246$ & $1200 \times 1600$ & $1944 \times 1536$ & $1944 \times 1536$ & $2400 \times 2400$ & $1350 \times 1350$ \\
\hline Dimensions $\left(\mathrm{cm}^{2}\right)$ & $2.49 \times 3.3$ & $2.7 \times 3.6$ & $14.5 \times 11.5$ & $14.5 \times 11.5$ & $12 \times 12$ & $5.4 \times 5.4$ \\
\hline MTF@10\% (cycles/mm) & 10.4 & 10.9 & 7.7 & 4.4 & 9 & 4.1 \\
\hline MTF@50\% (cycles/mm) & 3.6 & 3.8 & 2.7 & 1.3 & 2.7 & 1.3 \\
\hline
\end{tabular}

Horizontal and vertical 1D NNPS values, along with the radial average NNPS are depicted in Figure 5 for $28.1 \mu \mathrm{Gy}$ exposure, and in Figure 6 for $48.3 \mu \mathrm{Gy}$. The points isotropy (i.e., similarity of the magnitudes between axes) for both exposures varies through the whole range of spatial frequencies; from negligible differences, up to an order of magnitude at $10.1-10.6$ cycles $/ \mathrm{mm}$, where the vertical component exhibits a peak, probably due to remnant row-to-row FPN.

As expected, there is a tendency of NNPS decrease as the exposure increases. This is due to the fact that, with higher exposures the signal increase exceeds the noise increase (due to Poisson distribution in the detection of input X-rays). For example, at the exposure level of 28.1 the absolute NNPS value is $3.45 \times 10^{-6}$ at 0.78 cycles $/ \mathrm{mm}$, whereas the corresponding value for the exposure level of 48.3 is $1.84 \times 10^{-6}$.

In Figure 7 is drawn the DQE in both exposures of our experiments. Given that the MTF between $28.1 \mu \mathrm{Gy}$ and $48.3 \mu \mathrm{Gy}$ did not show any great differences, whereas the NNPS showed a tendency to decrease with increasing exposure, an analogous behavior is expected for the DQE curve, i.e., increased values with higher exposures. This is confirmed by the graph. Besides, the curves have similar shape, since the frequency composition is generally not affected by the exposure [53]; any shape discrepancies should be attributed to the inherent non-linearity of CMOS APS detectors, remnant FPN and electronic noise.

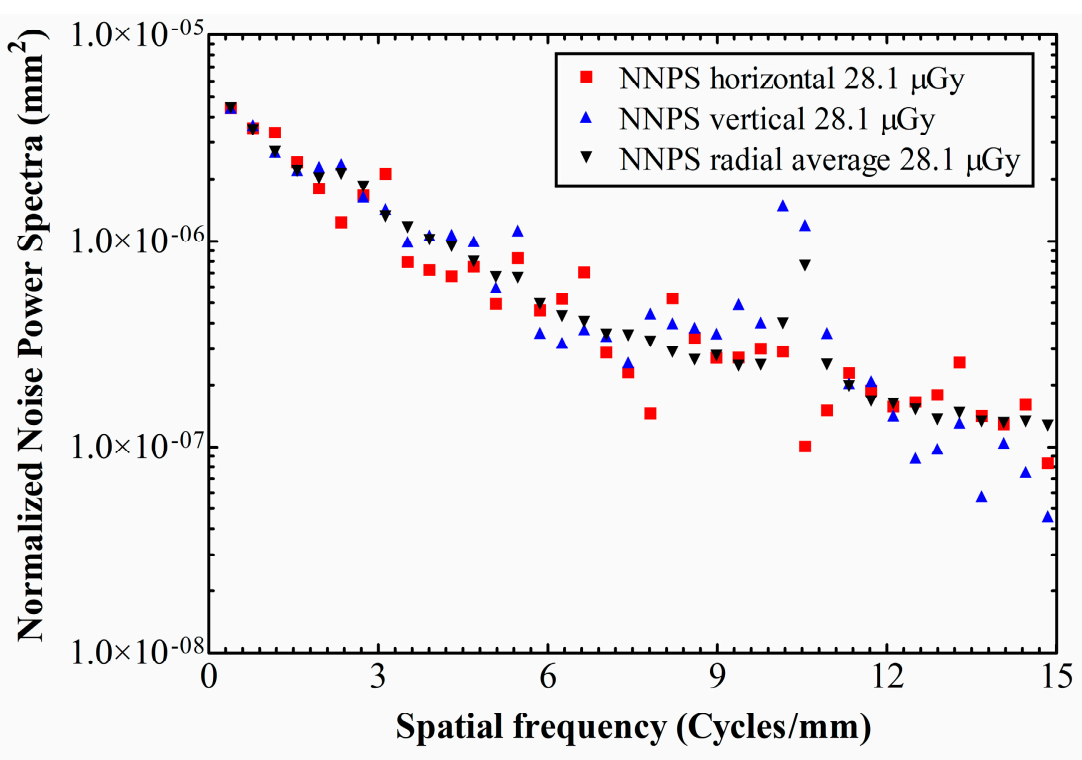

Figure 5. NNPS values at an exposure level of $28.1 \mu \mathrm{Gy}$. 


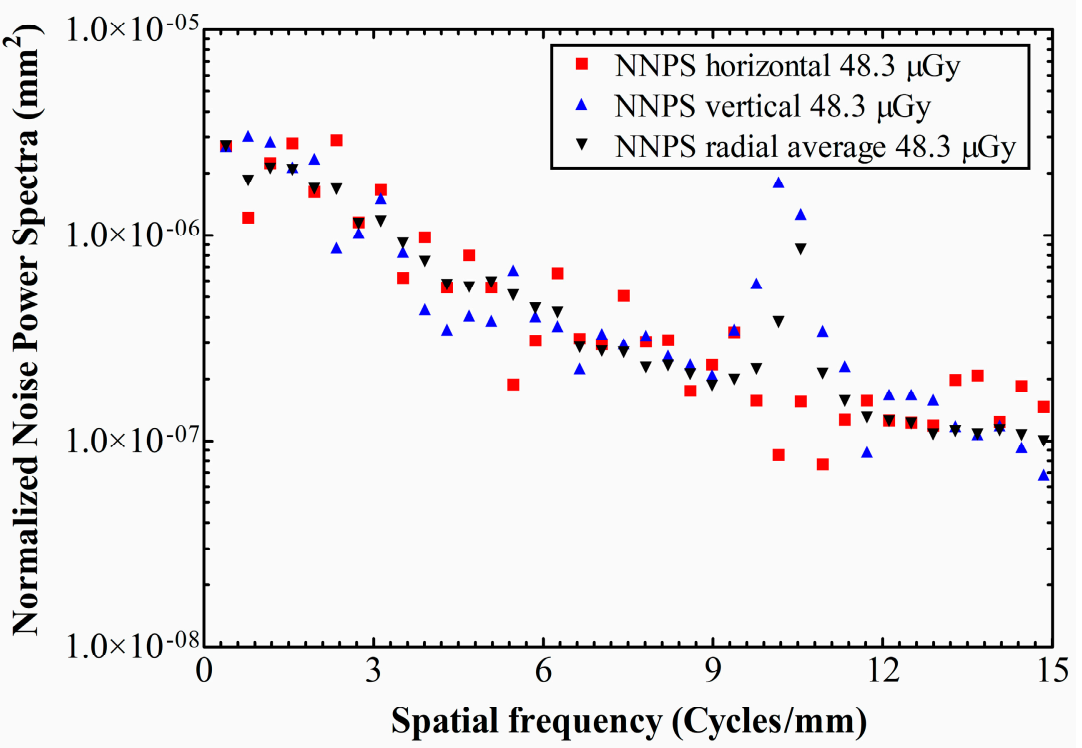

Figure 6. NNPS values at an exposure level of $48.3 \mu \mathrm{Gy}$.

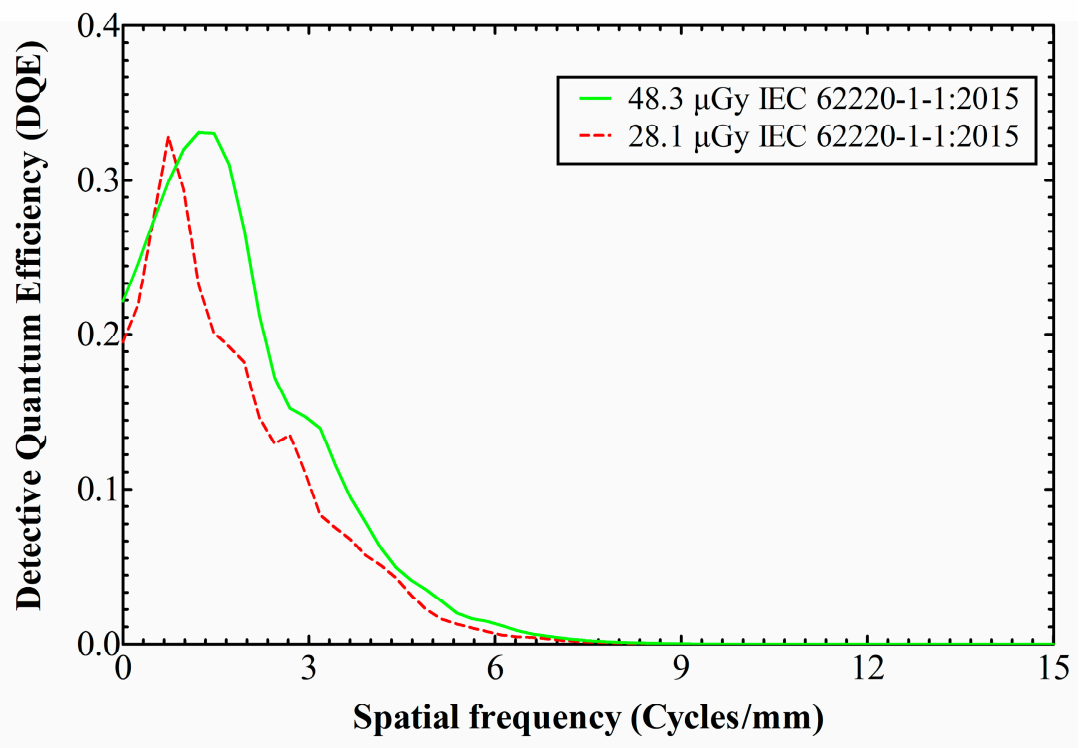

Figure 7. DQE curves for both the examined exposures.

Other than that, the DQE peaks at 1 cycle/mm and then drops with spatial frequency, indicating that the SNR impinging on the detector is transferred less efficiently towards its output, as the spatial frequency increases. Under $\sim 1$ cycle/mm the NNPS levels are high enough to restrain the DQE values. Subsequently, the NNPS levels fall rapidly (Figures 5 and 6; logarithmic y-axes), thus letting the DQE values to build up. For the $48.3 \mu \mathrm{Gy}$ exposure, at the lowest spatial frequency limit the DQE starts from $\mathrm{DQE}(0)=0.22$, then peaks at $\operatorname{DQE}(1.3)=0.33$ and falls to 0.1 at 3.6 cycles $/ \mathrm{mm}$. As for the $28.1 \mu \mathrm{Gy}$ exposure, the DQE starts from $\operatorname{DQE}(0)=0.19$, then peaks at $\operatorname{DQE}(0.7)=0.33$ and falls to 0.1 at 3.0 cycles $/ \mathrm{mm}$.

As an indication of the present detector's imaging characteristics in the NDT context, a sample radiography is displayed in Figure 8. The filament coil (0.2 mm thickness) is clearly discernible. The sample lamp (halogen type; Geyer G4 12 V 28 W, Chalkida, Greece) was placed on the detector's surface and an exposure was taken at $70 \mathrm{kVp} 20 \mathrm{mAs}$, using the same SDD as of the measurements, i.e., practically without geometric magnification. 


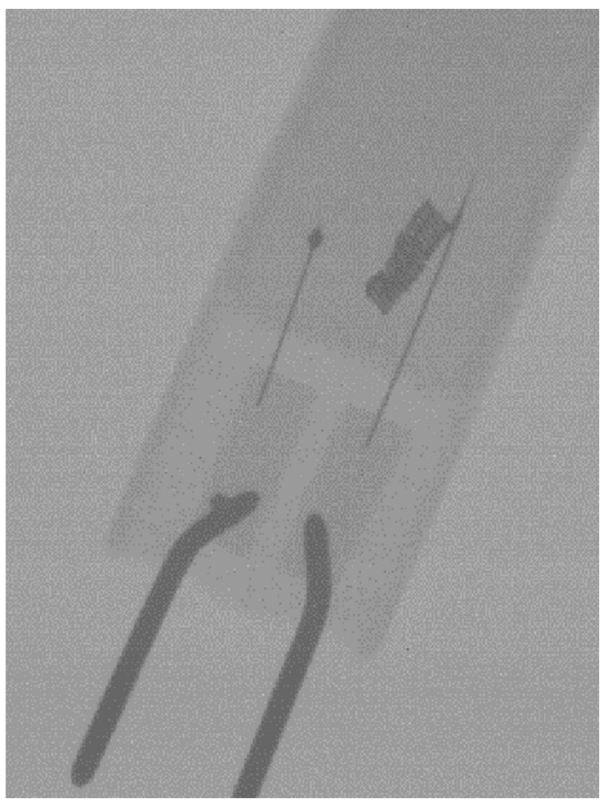

Figure 8. Halogen lamp radiography on the Remote RadEye HR (70 kVp 20 mAs).

\section{Potential Application for Dual-Energy Imaging}

The Min- $\mathrm{R}$ screen of the present work is made of $\mathrm{Gd}_{2} \mathrm{O}_{2} \mathrm{~S}: \mathrm{Tb}$, one of the most commonly used scintillating materials coupled to digital imagers. Of its advantageous characteristics are the large effective atomic number $\left(Z_{\text {eff }}=60\right)$ and its density $\left(7.3 \mathrm{~g} / \mathrm{cm}^{3}\right)$, that both favor X-ray absorption. It also exhibits a high light conversion efficiency $(19 \%)$ and a high light yield (60,000 photons/MeV) [54].

In dual-energy detectors, $\mathrm{X}$-rays of different energies are absorbed selectively by two scintillators of the appropriate properties, thus improving the capability to image different composition materials. DE mammography for instance, has been proven to suppress the contrast between overlapping tissues, enhancing in this way the detectability of micro-calcifications and masses $[29,55,56]$. In the industrial context on the other hand, dual-energy imaging can provide quantitative information of the interior structure of composite materials. Examples are flaw detection of welds or complex geometry parts, luggage inspection, food industry samples [8-10].

The pair $\mathrm{ZnSe}: \mathrm{Te}$ and $\mathrm{CdWO}_{4}$ is commonly used in such arrays, with $\mathrm{ZnSe}: \mathrm{Te}$ (low $Z_{\text {eff }}$ and density) being the first layer to absorb the lower-energy photons and $\mathrm{CdWO}_{4}$ (high $\mathrm{Z}_{\text {eff }}$ and density) detecting the $\mathrm{X}$-rays that traversed the first layer. Comparing the properties of $\mathrm{Gd}_{2} \mathrm{O}_{2} \mathrm{~S}: \mathrm{Tb}$ and $\mathrm{CdWO}_{4}$, one is becoming aware of the certain properties they have in common. Namely, $\mathrm{CdWO}_{4}$ has effective atomic number 64.2 , density $7.9 \mathrm{~g} / \mathrm{cm}^{3}$ and light yield 19,700 photons / $\mathrm{MeV}$ [57]. Its emission spectrum peaks at $495 \mathrm{~nm}$, therefore it is well differentiated from that of ZnSe:Te (640 nm peak). Both spectra though, stand within the present imager's spectral sensitivity range ( 0.97 matching factor with ZnSe:Te) [58,59]. These results indicate high potential for the two phosphors, $\mathrm{Gd}_{2} \mathrm{O}_{2} \mathrm{~S}: \mathrm{Tb}$ and $\mathrm{ZnSe}: \mathrm{Te}$, to be considered as the high- and low-energy elements, respectively, of a dual-energy flat imager.

Such a material combination has been used by Altman et al. [60] for a dual-energy computed tomography (DECT) application. Nevertheless, in this approach the sensing photodetectors are placed next to every row of phosphor pair, i.e., the light detection takes place through the scintillators' side surfaces. This means an insensitive area of $0.125 \mathrm{~mm}$ thickness next to each phosphor row, as well as the necessity to radiation-shield these photodetectors [60].

The previous version of the examined detector, also having $\mathrm{Gd}_{2} \mathrm{O}_{2} \mathrm{~S}: \mathrm{Tb}$ phosphor, has been used in dual-energy breast imaging resulting in the detectability of $150 \mu \mathrm{m}$ thick calcifications. The small pixel pitch allowed post processing in the final images and thus a $93 \mu \mathrm{m}$ thick calcification was visible [55]. Considering that systems used in digital 
mammography can detect calcifications as small as $130 \mu \mathrm{m}$ [61], such a detector has the potential to be used in dual-energy imaging. The same detector was also used in a study for characterization between malignant and benign calcifications. Based on the results of the study, such characterization could be accomplished for calcification thicknesses of $300 \mu \mathrm{m}$ or higher [29]. Although compared to the previous version of RadEye HR the new version somehow has lower resolution $(4.8 \%$ and $5.5 \%$ differences at $10 \%$ and $50 \%$ of the MTF, respectively; please see Table 1), such a slight reduction cannot substantially affect its diagnostic performance.

In conclusion, for those applications requesting low to medium diagnostic $X$-ray energies, the scintillator pair $\mathrm{Gd}_{2} \mathrm{O}_{2} \mathrm{~S}: \mathrm{Tb}$ and $\mathrm{ZnSe}$ :Te with the present CMOS imager could be considered as a possible DE array. Diverse approaches have been proposed, yet none of which utilizing these materials in a 2-D panel configuration, to the best of our knowledge. One could be similar to the dual-energy dual-color approach of Maier et al. [62]; the two phosphors are layered together, and the signal is differentiated by means of their different emission wavelengths and an optical layout consisting of a dichroic mirror and lenses. Another could be analogous to the Han et al. [63] solution of a sandwich detector; two $\mathrm{Gd}_{2} \mathrm{O}_{2} \mathrm{~S}$ :Tb phosphors of different thicknesses are separated by an optically-opaque, radio-translucent foil and a CMOS is coupled on each side.

\section{Conclusions}

The present NDT CMOS-based detector exhibits excellent linearity across the examined exposure range and a higher gain factor compared to the older $22.5 \mu \mathrm{m}$ CMOS. The MTF calculated according to the IEC 2003 standard is in close agreement with the CMOS manufacturer's MTF curve, whereas the MTF calculated with IEC 2015 is lower in the higher frequency range, due to the averaging method of IEC 2003 that leads to an overestimation of the MTF. MTF values are higher compared to other commercial CMOS detectors. However, the previous, discontinued, version of the detector under investigation performs slightly better in terms of the MTF, despite its coarser pixel pitch $(22.5 \mu \mathrm{m}$ instead of $20.0 \mu \mathrm{m}$ ). Regarding the detector's phosphor material, $\mathrm{Gd}_{2} \mathrm{O}_{2} \mathrm{~S}: \mathrm{Tb}$, and based on its properties' pertinence to those of $\mathrm{CdWO}_{4}$, dual-energy application is discussed. Specifically, the present CMOS with its $\mathrm{Gd}_{2} \mathrm{O}_{2} \mathrm{~S}: \mathrm{Tb}$ scintillator, in conjunction with ZnSe: Te, appears as a promising candidate for a dual-energy flat imager aimed at the NDT and medical fields.

Author Contributions: Conceptualization, C.M. and G.F.; methodology, C.M., D.L., I.V., V.K., A.B., and N.M.; software, V.K., and N.M.; validation, C.M. and A.K.; formal analysis, C.M., D.L., and G.F.; investigation, C.M., G.F., D.L., A.K., and I.V.; resources, A.B.; data curation, C.M., D.L., N.M., and V.K.; writing—original draft preparation, D.L. and C.M.; writing—review and editing, D.L., C.M., A.K., and I.V.; visualization, I.V.; supervision, C.M.; project administration, C.M., and G.F.; funding acquisition, I.V. All authors have read and agreed to the published version of the manuscript.

Funding: The APC was funded by the Special Account for Research Grants, of the University of West Attica, Greece.

Institutional Review Board Statement: Not applicable.

Informed Consent Statement: Not applicable.

Data Availability Statement: Data is contained within the article..

Conflicts of Interest: The authors declare no conflict of interest.

\section{References}

1. Zhao, C.; Kanicki, J.; Konstantinidis, A.C.A.C.; Patel, T. Large area CMOS active pixel sensor X-ray imager for digital breast tomosynthesis: Analysis, modeling, and characterization. Med. Phys. 2015, 42, 6294-6308. [CrossRef]

2. Konstantinidis, A.C.; Szafraniec, M.B.; Speller, R.D.; Olivo, A. The Dexela 2923 CMOS X-ray detector: A flat panel detector based on CMOS active pixel sensors for medical imaging applications. Nucl. Instrum. Methods Phys. Res. A 2012, 689, 12-21. [CrossRef] 
3. Zhao, C.; Konstantinidis, A.C.; Zheng, Y.; Anaxagoras, T.; Speller, R.D.; Kanicki, J. 50 m pixel pitch wafer-scale CMOS active pixel sensor X-ray detector for digital breast tomosynthesis. Phys. Med. Biol. 2015, 60, 8977-9001. [CrossRef]

4. Zhao, C.; Kanicki, J. Amorphous In-Ga-Zn-O thin-film transistor active pixel sensor X-ray imager for digital breast tomosynthesis. Med. Phys. 2014, 41, 091902. [CrossRef]

5. Cabello, J.; Bailey, A.; Kitchen, I.; Clark, A.; Crooks, J.; Halsall, R.; Key-Charriere, M.; Martin, S.; Prydderch, M.; Turchetta, R.; et al. Digital autoradiography using CCD and CMOS imaging technology. In Proceedings of the 2006 IEEE Nuclear Science Symposium Conference Record (NSS/MIC 2006), San Diego, CA, USA, 29 October-4 November 2006; Volume 4, pp. $2607-2612$.

6. Pham, T.N.; Finck, C.; Marchand, P.; Brasse, D.; Boisson, F.; Laquerriere, P. 18F autoradiography using pixelated CMOS technology. In Proceedings of the 2017 15th IEEE International New Circuits and Systems Conference (NEWCAS 2017), Strasbourg, France, 25-28 June 2017; pp. 41-44.

7. O'Connell, D.W.; Morgan, K.S.; Ruben, G.; Schaff, F.; Croton, L.C.P.; Buckley, G.A.; Paganin, D.M.; Uesugi, K.; Kitchen, M.J. Photon-counting, energy-resolving and super-resolution phase contrast X-ray imaging using an integrating detector. Opt. Express 2020, 28, 7080-7094. [CrossRef]

8. Kupsch, A.; Hentschel, M.; Lange, A.; Bruno, G.; Müller, B. Direct X-ray Refraction of Micro Structures. In Proceedings of the 11th European Conference on Non-Destructive Testing (ECNDT 2014), Prague, Czech Republic, 6-10 October 2014.

9. Mishurova, T.; Artzt, K.; Haubrich, J.; Requena, G.; Bruno, G. New aspects about the search for the most relevant parameters optimizing SLM materials. Addit. Manuf. 2019, 25, 325-334. [CrossRef]

10. Prosch, A.; Larson, B. Real Time Radiography NDT Course Booklet. Available online: https: / / www.nde-ed.org/TeachingResources/ Downloadable/Real-Time\%20Radiography/Real-time\%20Radiography\%20Booklet.htm (accessed on 12 February 2021).

11. Nikl, M. Scintillation detectors for X-rays. Meas. Sci. Technol. 2006, 17, R37-R54. [CrossRef]

12. A Elbakri, I.; McIntosh, B.J.; Rickey, D.W. Physical characterization and performance comparison of active- and passive-pixel CMOS detectors for mammography. Phys. Med. Biol. 2009, 54, 1743-1755. [CrossRef] [PubMed]

13. Michail, C.M.; Spyropoulou, V.A.; Fountos, G.P.; Kalyvas, N.I.; Valais, I.G.; Kandarakis, I.S.; Panayiotakis, G.S. Experimental and Theoretical Evaluation of a High Resolution CMOS Based Detector Under X-ray Imaging Conditions. IEEE Trans. Nucl. Sci. 2010, 58, 314-322. [CrossRef]

14. Konstantinidis, A.; Anaxagoras, T.; Esposito, M.; Allinson, N.; Speller, R. DynAMITe: A prototype large area CMOS APS for breast cancer diagnosis using X-ray diffraction measurements. SPIE Med Imaging 2012, 8313, 83135. [CrossRef]

15. Teledyne CCD290-99 Product Datasheet. Available online: https://www.teledyne-e2v.com/markets/space/astronomy-imaging/ ccd290-99/ (accessed on 12 February 2021).

16. E Bohndiek, S.; Cook, E.J.; Arvanitis, C.D.; Olivo, A.; Royle, G.J.; Clark, A.T.; Prydderch, M.L.; Turchetta, R.; Speller, R.D. A CMOS active pixel sensor system for laboratory- based X-ray diffraction studies of biological tissue. Phys. Med. Biol. 2008, 53, 655-672. [CrossRef]

17. Rowlands, J.A.; Yorkston, J. Flat Panel Detectors for Digital Radiography (Part I, Chapter 4). In Handbook of Medical Imaging. Volume 1: Physics and Psychophysics; Beutel, J., Kundel, H.L., Van Metter, R.L., Eds.; SPIE Press: Bellingham, WA, USA, 2000; Volume 1, pp. 223-327. ISBN 978-0-8194-7772-9.

18. Konstantinidis, A.C.; Szafraniec, M.B.; Rigon, L.; Tromba, G.; Dreossi, D.; Sodini, N.; Liaparinos, P.F.; Naday, S.; Gunn, S.; McArthur, A.; et al. X-ray Performance Evaluation of the Dexela CMOS APS X-ray Detector Using Monochromatic Synchrotron Radiation in the Mammographic Energy Range. IEEE Trans. Nucl. Sci. 2013, 60, 3969-3980. [CrossRef]

19. Gopinath, D.; Soman, M.; Holland, A.; Keelan, J.; Hall, D.; Holland, K.; Colebrook, D. Soft X-ray radiation damage in EM-CCDs used for Resonant Inelastic X-ray Scattering. J. Instrum. 2018, 13, C02027. [CrossRef]

20. Adams, R.; Zboray, R. Gamma radiography and tomography with a CCD camera and Co-60 source. Appl. Radiat. Isot. 2017, 127, 82-86. [CrossRef]

21. Arvanitis, C.D.; Bohndiek, S.E.; Royle, G.; Blue, A.; Liang, H.X.; Clark, A.; Prydderch, M.; Turchetta, R.; Speller, R. Empirical electro-optical and X-ray performance evaluation of CMOS active pixels sensor for low dose, high resolution X-ray medical imaging. Med. Phys. 2007, 34, 4612-4625. [CrossRef]

22. Ahn, J.; Moon, C.-R.; Kim, B.; Lee, K.; Kim, Y.; Lim, M.; Lee, W.; Park, H.; Moon, K.; Yoo, J.; et al. Advanced Image Sensor Technology for Pixel Scaling down toward $1.0 \mu \mathrm{m}$ (Invited). In Proceedings of the 2008 IEEE International Electron Devices Meeting, San Francisco, CA, USA, 15-17 December 2008; pp. 1-4.

23. Asakura, K.; Hayashida, K.; Hanasaka, T.; Kawabata, T.; Yoneyama, T.; Okazaki, K.; Ide, S.; Noda, H.; Matsumoto, H. X-ray imaging polarimetry with a 2.5- $\mu \mathrm{m}$ pixel CMOS sensor for visible light at room temperature. J. Astron. Telesc. Instrum. Syst. 2019, 5, 035002. [CrossRef]

24. Zhang, L.; Jin, Y.; Lin, L.; Li, J.; Du, Y. The Comparison of CCD and CMOS Image Sensors. In Proceedings of the SPIE, Proceedings of 2008 International Conference on Optical Instruments and Technology: Advanced Sensor Technologies and Applications, Beijing, China, 16-19 November 2008; Wang, A., Liao, Y., Song, A., Ishii, Y., Fan, X., Eds.; Curran Associates, Inc.: Red Hook, NY, USA, 2009.

25. Olbinado, M.P.; Just, X.; Gelet, J.-L.; Lhuissier, P.; Scheel, M.; Vagovic, P.; Sato, T.; Graceffa, R.; Schulz, J.; Mancuso, A.; et al. MHz frame rate hard X-ray phase-contrast imaging using synchrotron radiation. Opt. Express 2017, 25, 13857-13871. [CrossRef]

26. Teledyne Lince11M CMOS Sensor. Available online: https://imaging.teledyne-e2v.com/products/2d-cmos-image-sensors/ lince/lince-11m/ (accessed on 23 November 2020). 
27. Kuroda, R.; Sugawa, S. Cameras with On-chip Memory CMOS Image Sensors. In The Micro-World Observed by Ultra High-Speed Cameras; Springer International Publishing: New York, NY, USA, 2018; pp. 103-124.

28. Koukou, V.; Martini, N.; Fountos, G.; Michail, C.; Bakas, A.; Oikonomou, G.; Kandarakis, I.; Nikiforidis, G. Application of a dual energy X-ray imaging method on breast specimen. Results Phys. 2017, 7, 1634-1636. [CrossRef]

29. Martini, N.; Koukou, V.; Fountos, G.; Michail, C.; Bakas, A.; Kandarakis, I.; Speller, R.; Nikiforidis, G. Characterization of breast calcification types using dual energy X-ray method. Phys. Med. Biol. 2017, 62, 7741-7764. [CrossRef] [PubMed]

30. Teledyne Remote RadEye HR. Available online: https://www.teledynedalsa.com/en/products/imaging/industrial-X-ray/ remote-radeye-hr/ (accessed on 31 January 2021).

31. Cho, M.K.; Kim, H.K.; Graeve, T.; Yun, S.M.; Lim, C.H.; Cho, H.; Kim, J.-M. Measurements of X-ray Imaging Performance of Granular Phosphors With Direct-Coupled CMOS Sensors. IEEE Trans. Nucl. Sci. 2008, 55, 1338-1343. [CrossRef]

32. Konstantinidis, A. Evaluation of Digital X-ray Detectors for Medical Imaging Applications. PhD Thesis, University College London, London, UK, 2011.

33. Michail, C.; Valais, I.; Martini, N.; Koukou, V.; Kalyvas, N.; Bakas, A.; Kandarakis, I.; Fountos, G. Determination of the detective quantum efficiency (DQE) of CMOS/CsI imaging detectors following the novel IEC 62220-1-1:2015 International Standard. Radiat. Meas. 2016, 94, 8-17. [CrossRef]

34. International Electrotechnical Commission IEC 62220-1:2003 Medical Electrical Equipment-Characteristics of Digital X-ray Imaging Devices-Part 1: Determination of the Detective Quantum Efficiency; IEC: Geneva, Switzerland, 2003.

35. International Electrotechnical Commission IEC 62220-1-1:2015 Medical Electrical Equipment: Characteristics of Digital X-ray Imaging Devices. Part 1-1; IEC: Geneva, Switzerland, 2015; ISBN 978-2-8322-2389-5.

36. Donini, B.; Rivetti, S.; Lanconelli, N.; Bertolini, M. Free software for performing physical analysis of systems for digital radiography and mammography. Med. Phys. 2014, 41, 051903. [CrossRef]

37. ImageJ, An Open Platform for Scientific Image Analysis. Available online: https://imagej.net/Welcome (accessed on 12 February 2021).

38. Neitzel, U.; Günther-Kohfahl, S.; Borasi, G.; Samei, E. Determination of the detective quantum efficiency of a digital X-ray detector: Comparison of three evaluations using a common image data set. Med. Phys. 2004, 31, 2205-2211. [CrossRef]

39. Samei, E.; Flynn, M.J.; Reimann, D.A. A method for measuring the presampled MTF of digital radiographic systems using an edge test device. Med. Phys. 1998, 25, 102-113. [CrossRef]

40. Kandarakis, I.; Cavouras, D.; Sianoudis, I.; Nikolopoulos, D.; Episkopakis, A.; Linardatos, D.; Margetis, D.; Nirgianaki, E.; Roussou, M.; Melissaropoulos, P.; et al. On the response of $\mathrm{Y}_{3} \mathrm{Al}_{5} \mathrm{O}_{12}$ :Ce (YAG:Ce) powder scintillating screens to medical imaging X-rays. Nucl. Instrum. Methods Phys. Res. A 2005, 538, 615-630. [CrossRef]

41. Dobbins III, J.T. Image Quality Metrics for Digital Systems (Part I, Chapter 3). In Handbook of Medical Imaging. Volume 1: Physics and Psychophysics; Beutel, J., Kundel, H.L., Van Metter, R.L., Eds.; SPIE Press: Bellingham, WA, USA, 2000; Volume 1, pp. 161-222. ISBN 978-0-8194-7772-9.

42. Michail, C.; Valais, I.; Seferis, I.; Kalyvas, N.; Fountos, G.; Kandarakis, I. Experimental measurement of a high resolution CMOS detector coupled to CsI scintillators under X-ray radiation. Radiat. Meas. 2015, 74, 39-46. [CrossRef]

43. Fountos, G.P.; Michail, C.M.; Zanglis, A.; Samartzis, A.; Martini, N.; Koukou, V.; Kalatzis, I.; Kandarakis, I.S. A novel easy-to-use phantom for the determination of MTF in SPECT scanners. Med. Phys. 2012, 39, 1561. [CrossRef] [PubMed]

44. Michail, C. Image Quality Assessment of a CMOS $/ \mathrm{Gd}_{2} \mathrm{O}_{2} \mathrm{~S}$ :Pr,Ce,F X-ray Sensor. J. Sens. 2015, 2015, 1-6. [CrossRef]

45. Seferis, I.; Michail, C.; Valais, I.; Fountos, G.; Kalyvas, N.; Stromatia, F.; Oikonomou, G.; Kandarakis, I.; Panayiotakis, G. On the response of a europium doped phosphor-coated CMOS digital imaging detector. Nucl. Instrum. Methods Phys. Res. A 2013, 729, 307-315. [CrossRef]

46. Chan, H.-P.; Doi, K. Radiation dose in diagnostic radiology: Monte Carlo simulation studies. Med Phys. 1984, 11, 480-490. [CrossRef]

47. Marshall, N.W. Detective quantum efficiency measured as a function of energy for two full-field digital mammography systems. Phys. Med. Biol. 2009, 54, 2845-2861. [CrossRef]

48. Howansky, A.; Mishchenko, A.; Lubinsky, A.R.; Zhao, W. Comparison of CsI:Tl and $\mathrm{Gd}_{2} \mathrm{O}_{2} \mathrm{~S}: \mathrm{Tb}$ indirect flat panel detector X-ray imaging performance in front- and back-irradiation geometries. Med. Phys. 2019, 46, 4857-4868. [CrossRef]

49. Choi, S.; Seo, C.-W.; Cha, B.K. Effect of Filtered Back-Projection Filters to Low-Contrast Object Imaging in Ultra-High-Resolution (UHR) Cone-Beam Computed Tomography (CBCT). Sensors 2020, 20, 6416. [CrossRef]

50. Schulze, R.K.W.; I Doering, C. Simple computation of the approximated modulation transfer function (MTF) using spreadsheetsoftware: Method and evaluation in five maxillofacial CBCT-devices. Dentomaxillofacial Radiol. 2019, 48, 20180350. [CrossRef]

51. Hamamatsu Flat Panel Sensor C9732DK-11. Available online: https://www.hamamatsu.com/eu/en/product/type/C9732DK11/index.html (accessed on 31 January 2021).

52. Bohndiek, S.E.; Blue, A.; Cabello, J.; Clark, A.T.; Guerrini, N.; Evans, P.M.; Harris, E.J.; Konstantinidis, A.; Maneuski, D.; Osmond, J.; et al. Characterization and Testing of LAS: A Prototype 'Large Area Sensor' With Performance Characteristics Suitable for Medical Imaging Applications. IEEE Trans. Nucl. Sci. 2009, 56, 2938-2946. [CrossRef]

53. Monnin, P.; Gutierrez, D.; Bulling, S.; Guntern, D.; Verdun, F.R. A comparison of the performance of digital mammography systems. Med. Phys. 2007, 34, 906-914. [CrossRef] 
54. Litichevskyi, V.; Galkin, S.; Lalayants, O.; Voronkin, E.; Breslavskiy, I.; Tretiak, S.; Kosinov, N. Scintillation Panels Based on Zinc Selenide and Oxide Scintillators. Funct. Mater. 2011, 18, 391-397.

55. Koukou, V.; Martini, N.; Fountos, G.; Michail, C.; Sotiropoulou, P.; Bakas, A.; Kalyvas, N.; Kandarakis, I.; Speller, R.; Nikiforidis, G. Dual energy subtraction method for breast calcification imaging. Nucl. Instrum. Methods Phys. Res. A 2017, 848, 31-38. [CrossRef]

56. Martini, N.; Koukou, V.; Michail, C.; Fountos, G. Dual Energy X-ray Methods for the Characterization, Quantification and Imaging of Calcification Minerals and Masses in Breast. Cryst. 2020, 10, 198. [CrossRef]

57. Lecoq, P.; Gektin, A.; Korzhik, M. Inorganic Scintillators for Detector Systems: Physical Principles and Crystal Engineering; Springer: Berlin, Germany, 2006; ISBN 978-3-540-27766-8.

58. Linardatos, D.; Konstantinidis, A.; Valais, I.; Ninos, K.; Kalyvas, N.; Bakas, A.; Kandarakis, I.; Fountos, G.; Michail, C. On the Optical Response of Tellurium Activated Zinc Selenide ZnSe:Te Single Crystal. Crystals 2020, 10, 961. [CrossRef]

59. Seferis, I.E.; Michail, C.; Zeler, J.; Kalyvas, N.; Valais, I.; Fountos, G.; Bakas, A.; Kandarakis, I.; Zych, E.; Panayiotakis, G.S. Detective quantum efficiency (DQE) of high X-ray absorption Lu2O3:Eu thin screens: The role of shape and size of nano- and micro-grains. Appl. Phys. A 2018, 124, 604. [CrossRef]

60. Altman, A.; Shapiro, O.; Levene, S.; Wainer, N. Double Decker Detector for Spectral CT. US Patent US7968853B2, 28 June 2011.

61. Bick, U.; Diekmann, F. Digital Mammography; Springer: Heidelberg, Germany, 2010; ISBN 978-3-540-78449-4.

62. Maier, D.S.; Schock, J.; Pfeiffer, F. Dual-energy micro-CT with a dual-layer, dual-color, single-crystal scintillator. Opt. Express 2017, 25, 6924-6935. [CrossRef] [PubMed]

63. Han, J.C.; Kim, H.K.; Kim, N.W.; Yun, S.; Youn, H.; Kam, S.; Tanguay, J.; Cunningham, I.A. Single-shot dual-energy X-ray imaging with a flat-panel sandwich detector for preclinical imaging. Curr. Appl. Phys. 2014, 14, 1734-1742. [CrossRef] 